\title{
Comparing event-related and epoch analysis in blocked design fMRI
}

\author{
Andrea Mechelli, ${ }^{a,}{ }^{*}$ Rik N.A. Henson, ${ }^{\text {a,b }}$ Cathy J. Price, ${ }^{a}$ and Karl J. Friston ${ }^{a}$ \\ ${ }^{a}$ Wellcome Department of Imaging Neuroscience, Institute of Neurology, 12 Queen Square, London, WCIN 3BG, UK \\ ${ }^{\mathrm{b}}$ Institute of Cognitive Neuroscience, 17 Queen Square, London WC1N 3AR, UK
}

Received 23 April 2002; revised 6 August 2002; accepted 27 August 2002

\begin{abstract}
In this study we demonstrate that, even in blocked design fMRI, an event-related analysis may provide a more accurate model of the hemodynamic responses than an epoch-related analysis. This is because the temporal shape of the predicted response differs between the event-related and the epoch model, with the former reaching its peak sooner and returning to baseline later than the latter. We present data from a blocked design fMRI study of single word reading alternated with rest. Conventionally, such a design would be analyzed using an epoch analysis with boxcar regressors. However, here we used a combined model in which trials were modeled as both single events and epochs. This allowed us to estimate the variance in the BOLD signal that was explained by either the event-related or the epoch regressors having discounted the effect of the other. We found that, in a number of language regions, the event-related model explained changes in activity that were not accounted for by the epoch model. In addition, we show that the advantage of the event-related over epoch model was engendered by its early onset rather than its late offset, relative to the epoch model.
\end{abstract}

(C) 2003 Elsevier Science (USA). All rights reserved.

\section{Introduction}

In functional magnetic resonance imaging (fMRI), a distinction is made between event-related designs in which stimuli of different types are intermixed and blocked designs in which stimuli of the same type are presented in blocks. Effects of interest in blocked designs are usually modeled with some form of boxcar regressor convolved with a synthetic hemodynamic response function (HRF). Implicit in this model is the assumption that steady-state synaptic activity and hemodynamics are attained within each block. In contrast, effects of interest in event-related designs are modeled by convolving each trial onset (i.e., a stick function) with a synthetic HRF. Here the hemodynamic responses to stimulus-induced neuronal transients are modeled without assuming constant within-block activity.

Crucially, event-related models can also be used in the context of blocked designs (Price et al., 1999). However, they can differ from epoch models with respect to the temporal form or shape of the predicted hemodynamic re-

\footnotetext{
* Corresponding author. Fax: +0044-020-78131420.

E-mail address: andream@fil.ion.ucl.ac.uk (A. Mechelli).
}

sponse. Fig. 1 illustrates differences in onset and offset latencies between epoch (solid line) and event-related (broken line) models, when the stimulus onset asynchrony (SOA) is $3 \mathrm{~s}$. It can be seen that, although the two models assume the same amount of integrated synaptic activity, the event-related model expresses higher frequencies than the epoch model (Fig. 1a). Once the assumed synaptic activity is convolved with the HRF (Fig. 1b), the two models predict differential hemodynamic responses, with the epoch model reaching its peak later and returning to baseline sooner than the event-related model (Fig. 1c). This corresponds to differential response onsets and offsets for the event-related and the epoch model. When the event-related model is orthogonalized with respect to the epoch model (Fig. 1d), effects that are modeled by the event-related but not by the epoch model are identified. It is apparent that the differences between the event-related and the epoch models arise primarily at the beginning and at the end of the block.

When the underlying hemodynamic response does not conform to the model adopted, residual (estimated error) variance increases and the sensitivity with which statistical inferences are drawn may be compromised. A standard event-related model should provide an accurate character- 
Neuronal Models
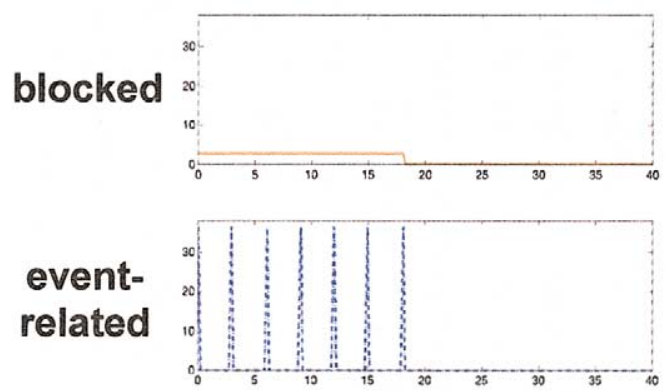

(a)
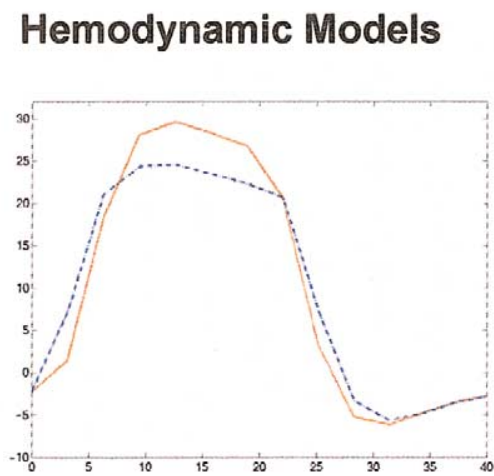

1
Time (s)

(c)
Time (s)
HRF

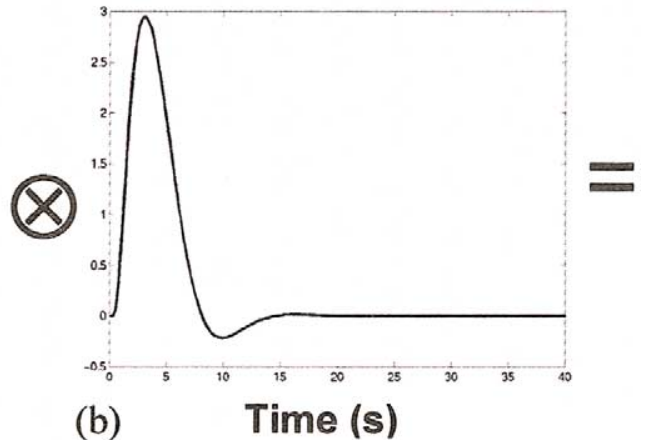

Event-Related - Epoch

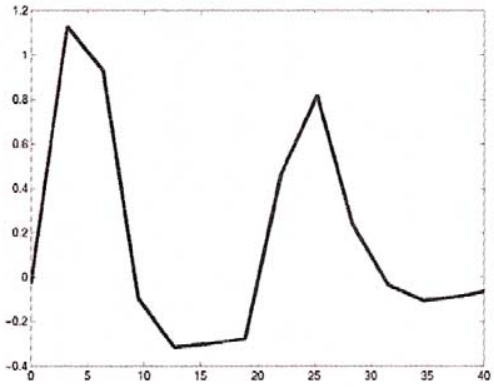

(d)

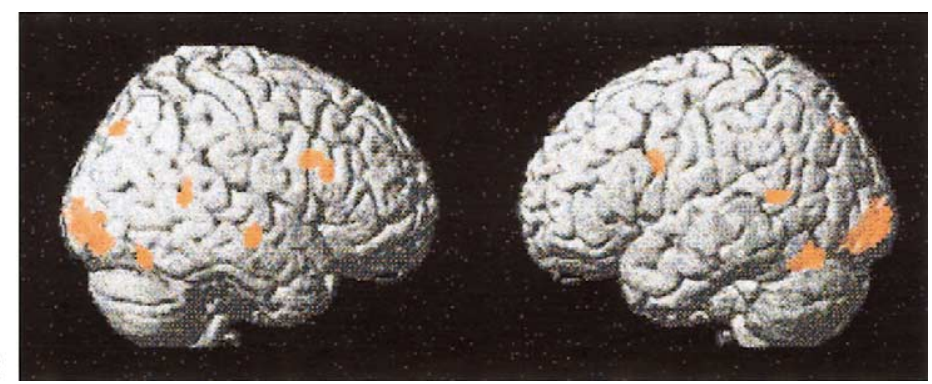

Fig. 1. Difference in onset and offset latencies between epoch (solid line) and event-related (broken line) models, when the SOA is $3 \mathrm{~s}$. (a) Neuronal models for epoch and event-related models. (b) The neuronal models are convolved with the HRF to predict the hemodynamic responses. (c) Differential hemodynamic responses predicted by the epoch and the event-related model. Although the area under curves is the same, the epoch model reaches its peak later and returns to baseline sooner than the event-related model. (d) When the epoch model is orthogonalized with respect to the event-related model, effects which are modeled by the event-related but not by the epoch model are identified. It can be seen that the event-related model explains changes in activity at the beginning and at the end of the block that are not accounted for by the epoch model.

Fig. 2. Regions that showed increased activity for reading relative to fixation that was explained by the event-related but not by the epoch model (Analysis 1). These include language areas such as left inferior fusiform, superior posterior temporal, and inferior frontal cortex (see Table 1 for details). These regions also showed an effect of reading relative to fixation in Mechelli et al. (2000).

ization of the latency of brain responses, even in the context of blocked design fMRI. We illustrate this empirically by presenting data from a blocked design fMRI study on single word reading alternated with rest. Conventionally, such a design would be analyzed using a boxcar regressor convolved with the HRF. However, here we used a combined model in which trials were modeled as both epochs and single events. This allowed us to estimate the variance in the BOLD signal that was explained by either the epoch or event-related model above and beyond the other. If the observed response conforms to a HRF convolved with a boxcar regressor, then the event-related model would not evidence an advantage over the epoch model. In contrast, if signal changes reflected the additive contribution of the individual evoked responses to each stimulus, then the event-related model would explain changes in activity more accurately than the epoch model. This would be expressed as a significant effect of reading relative to rest that is 
explained by the event-related but not by the epoch model. This is indeed what we found. In addition, we show that the advantage of the event-related related model results from its early onset rather then its late offset relative to the epoch model. The neurophysiological findings of this study were reported in Mechelli et al. (2000).

\section{Methods}

The study was approved by the National Hospital for Neurology and Institute of Neurology Medical Ethic's Committee.

\section{Subjects}

Informed consent was obtained from 6 right-handed volunteers (5 males), aged 20 to 34 (mean age of 24), with English as their first language.

\section{Task paradigm and stimuli}

Each subject was presented with blocks of words or pseudowords alternating with blocks of fixation (a cross in the middle of the screen). They were instructed to read each word and pseudoword silently as soon as it appeared on the screen. The variables were: (i) stimulus type (words and pseudowords) and (ii) stimulus rate (20, 40, and 60 words per minute, corresponding to an SOA of 3, 1.5, and $1 \mathrm{~s}$ ). Pseudowords (e.g., lenner) refer to letter strings that are not real words and do not have semantic representations but can be pronounced on the basis of sublexical spelling to sound relationships. Words were matched for frequency (Kucera and Francis, 1967), length, and number of syllables between blocks and were as regular as possible in terms of grapheme-phoneme conversion. Stimulus duration was $600 \mathrm{~ms}$. Each experimental condition was repeated 5 times in a counterbalanced order across subjects. Each block of stimuli lasted around $18 \mathrm{~s}$ and was followed by $18 \mathrm{~s}$ fixation. An eye tracker was used to ensure that the subjects kept their eyes open and attended to the stimuli.

\section{Data acquisition}

A $2 \mathrm{~T}$ Siemens VISION system (Siemens, Erlangen, Germany) was used to acquire both $T_{1}$ anatomical volume images $(1 \times 1 \times 1.5$-mm voxels $)$ and $T_{2}{ }^{*}$-weighted echoplanar images $(64 \times 643 \times 3-\mathrm{mm}$ pixels, TE $=40 \mathrm{~ms})$ with BOLD contrast. Each echoplanar image comprised 35 $1.8-\mathrm{mm}$ axial slices with a $1.2-\mathrm{mm}$ slice interval, giving a resolution of $3 \mathrm{~mm}$. A total of 366 volume images were obtained continuously with an effective repetition time (TR) of $3.15 \mathrm{~s}$ per volume, the first 6 volumes in each session being discarded to allow for $T_{1}$ equilibration effects. Stimulus presentation was arranged so that every $90 \mathrm{~ms}$ of peristimulus time was sampled equally over the session (see Price et al., 1999).

\section{Data analysis}

Data were analyzed using SPM99 (SPM99: Wellcome Department of Imaging Neuroscience, London, UK (http:// www.fil.ion.ucl.ac.uk). All volumes from each subject were realigned using the first as a reference and resliced (using sinc interpolation) adjusting for residual motion-related signal changes. A mean volume was created using the realigned volumes and the anatomical MRI was coregistered to it. This ensured that the functional and structural images were spatially aligned. The functional images were spatially normalized (Friston et al., 1995) with respect to the MNI305 template using nonlinear-basis functions. Functional data were spatially smoothed with a $6 \mathrm{~mm}$ full width at half-maximum, isotropic Gaussian kernel. Three statistical analyses were performed.

\section{Analysis 1: event-related and epoch regressors}

A combined model was constructed in which events were modeled as both epochs and single events. The resulting design comprised 6 "epoch" effects, in which trials were modeled as blocks using a boxcar regressor, and 6 "event-related" responses, in which the same trials were modeled as single events. Both were convolved with a HRF. This analysis identified effects of reading relative to fixation (using $t$ tests) that were explained by either model with the other acting as a nonorthogonal confound. However, this analysis did not determine whether the better fit of either model lay in the onset of the response (earlier in the event-related model), the offset of the response (later in the event-related model), or both (see Fig. 1d). This required two further analyses.

\section{Analysis 2: event-related, epoch, and first-stimulus regressors}

A combined model was constructed in which events were modeled as both epochs and single events, as in Analysis 1 . However, an additional regressor, representing the first stimulus in each block, was modeled using an individual delta function. The resulting design comprised 6 epoch regressors, 6 event-related regressors, in which the same trials were modeled as single events, and 6 "first stimulus" regressors, in which the first trial in each block was modeled as a single event. By modeling the first stimulus in each block as single event, most of the difference in latency onset between the event-related and the epoch model was discounted. Thus, the residual advantage of either model would be explaind by the differential offset of the expected epoch response (later in the event-related model). 
Table 1

\begin{tabular}{|c|c|c|c|c|c|c|}
\hline \multicolumn{7}{|c|}{ Reading $>$ fixation (event-related regressor only) } \\
\hline & & & & Analysis 1 & Analysis 2 & Analysis 3 \\
\hline \multicolumn{7}{|l|}{ Occipital } \\
\hline Left fusiform & -18 & -102 & 2 & 5.9 & 1.2 & 6.0 \\
\hline Right fusiform & 18 & -88 & -12 & 7.0 & 0.3 & 7.7 \\
\hline Left lingual & -8 & -96 & -2 & 6.2 & 0.2 & 7.1 \\
\hline Right middle occipital & 32 & -88 & -2 & 5.6 & 0.1 & 6.8 \\
\hline \multicolumn{7}{|l|}{ Temporal } \\
\hline Left posterior inferior temporal & -36 & -66 & -18 & 6.5 & 0.3 & 7.3 \\
\hline Right posterior inferior temporal & 38 & -66 & -18 & 5.6 & 0.2 & 7.1 \\
\hline Left superior temporal & -54 & -50 & 10 & 6.5 & 0.9 & 7.0 \\
\hline Right superior temporal & 62 & -48 & 10 & 5.8 & 2.7 & 5.6 \\
\hline Right middle temporal & 64 & -16 & -10 & 6.3 & 0.6 & 7.0 \\
\hline \multicolumn{7}{|l|}{ Parietal } \\
\hline Right angular gyrus & 24 & -80 & 42 & 6.0 & 0.6 & 6.7 \\
\hline \multicolumn{7}{|l|}{ Frontal } \\
\hline Right inferior frontal & 44 & 12 & 28 & 6.3 & 0.1 & 6.9 \\
\hline Left inferior frontal & -36 & 6 & 26 & 6.1 & 0.8 & 6.5 \\
\hline
\end{tabular}

Note. Effects of reading relative to fixation that were explained by the event-related but not by the epoch model (Analysis 1). These effects were no longer significant when the difference in the onset of the epoch and the event-related model was discounted (Analysis 2). In contrast, they were still significant when the late offset of the event-related model was discounted (Analysis 3). Significant effects (at $P<0.05$ corrected for multiple comparisons) are reported in bold.

Analysis 3: event-related, epoch, and last-stimulus regressors

A combined model was constructed in which events were modeled as both epochs and single events, as in Analysis 1, with an additional regressor modeling the last stimulus in each block. The resulting design comprised 6 epoch, 6 event-related and 6 "last stimulus" regressors. In this analysis, any residual advantage of either model would be explained by the differential onset of the predicted epoch response (earlier in the event-related model).

The data were high-pass-filtered using a set of discrete cosine basis functions with a cutoff period of $120 \mathrm{~s}$ : The temporal autocorrelations in the errors were estimated using a AR(1) + white noise model (Friston et al., 2002) and used to make the appropriate nonsphericity adjustment at the point of inference. Inferences were derived from a fixed effect analysis via $t$ tests of the event-related/epoch-related response versus baseline fixation, thresholded at $P<0.05$ (corrected for multiple comparisons) with an extent threshold of 15 voxels.

\section{Results}

When events were modeled as both epochs and single events (Analysis 1), activation for reading relative to fixation for the event-related regressor was found in a number of language regions (see Fig. 2 and Table 1 for details). In these regions, the event-related regressor explained changes in BOLD signal that were not accounted for by the epoch regressor. In contrast, activation for reading relative to fix- ation for the epoch regressor was not detected, even when lowering the statistical threshold to $P<0.001$ (uncorrected for multiple comparisons). This means that the epoch regressor did not explain changes in BOLD signal that were not accounted for by the event-related regressor. No deactivations relative to fixation were found for either regressor.

Activation for reading relative to fixation captured by the event-related regressor was observed for each stimulus rate and word type $(P<0.001$ uncorrected $)$. However, a greater event-related response was observed for reading 20 relative to 60 words per minute in left posterior inferior temporal ( $x$ $=-34, y=-68, z=-16 ; Z$-score $=4.9)$, right inferior fusiform $(x=16, y=-96, z=-10 ; Z$-score $=3.9)$, and left inferior frontal $(x=-40, y=6, z=22 ; Z$-score $=3.6)$ at an uncorrected level $(P<0.001)$. This trend was expected since the difference in latency between event-related and epoch regressors is a function of stimulus rate. This difference would disappear in the limit of very fast presentation rates because the epoch and event-related regressors would be the same.

When the first stimulus in each block was added to the model (Analysis 2), activation for reading relative to fixation using the event-related regressor only was no longer detected even when lowering the statistical threshold to $P<$ 0.001 (uncorrected for multiple comparisons) (see Table 1). In other words, the event-related regressor no longer explained changes in BOLD signal that were not accounted for by the epoch regressor. In contrast, when the last stimulus in each block was modeled (Analysis 3), activation for reading relative to fixation using the event-related regressor only was still significant, with $Z$-scores slightly higher than in Analysis 1 (see Table 1). 


\section{Discussion}

In the present study, we investigated the relative sensitivity of standard epoch and event-related analyses using a blocked design reading paradigm. In order to avoid anecdotal comparison of the contrast-specific statistics, which may be confounded by differential efficiency of response estimation for epoch and event-related models (see Mechelli et al., 2003), we used a combined model in which trials were characterized as both epochs and single events (Analysis 1). This allowed us to estimate directly the additional variance in the BOLD signal that was explained by either model using the extra sum of squares principle. We demonstrated that, in language regions, a significant amount of variance in the BOLD signal was accounted for by the event-related model that was not modeled by the epoch-related regressors. Although activation in these regions was reliably detected using an epoch model (see Mechelli et al., 2000), our study shows that an event-related approach may characterize the observed response with greater finesse, thereby maximizing sensitivity.

In order to determine whether the advantage of the eventrelated model lay in the early onset or the late offset of the modeled response, we also modeled the first and the last stimuli in each epoch (Analysis 2 and 3, respectively). When the early onset of the event-related model was discounted (Analysis 2), the event-related model no longer showed an advantage. In contrast, when the late offset of the event-related model was discounted (Analysis 3 ), the eventrelated model retained its ability to explain changes in BOLD signal that were not accounted for by the epoch model. This indicates that the advantage of the event-related model lies in its early onset rather than its late offset. One possibility for this asymmetry is that adaptation renders the stationary linear model for evoked responses less appropriate toward the end of a block. These results are consistent with the findings reported by Konishi et al. (2001), who detected transient responses during block transitions. It should be noted that the advantage of the event related model was preserved when the temporal derivatives were included in the epoch model, suggesting that the advantage of the event-related model was not simply due to a difference in latency but to a difference in the shape of the hemodynamic response.

While this work concentrates on the impact of the onset and offset differencies in event-related and epoch models, there are other reasons why the evoked BOLD responses to a train of stimuli may not conform to a boxcar regressor convolved with a HRF. For instance, for any sequence of repeated stimuli, the SOA may be too long, or the evoked hemodynamic response too transient, for a steady state to be attained. In either case, the response to a stimulus may have declined before the presentation of a subsequent stimulus. This will result in a periodic and dynamically modulated response which would be explained better by an eventrelated than an epoch model. Finally, the shape of the overall hemodynamic response may be affected by nonlinearities in the BOLD signal, especially for short SOAs or long epoch lengths (Mechelli et al., 2001). However, it is important to note that BOLD saturation effects as a function of stimulus rate cannot explain the advantage of eventrelated over epoch-related analyses. This is because we modeled each stimulus rate separately, allowing for any nonlinear relationship between rate of presentation and response (see Friston et al., 1998, for a full discussion of this in relation to generalized convolution models).

A question of interest is whether the findings reported here can be generalized to different cognitive paradigms. In fact it is possible that, under other circumstances, an epoch model may provide greater sensitivity than an event-related model. For instance, if the evoked hemodynamic response rises slowly or quickly returns to baseline, an epoch model may explain the response profile better than an event-related model. This is because epoch models reach their peak later and return to baseline earlier than event-related models, see Fig. 1.

In summary, we have demonstrated that an event-related model may characterize the form (particularly the onset) of the observed hemodynamic response better than an epochrelated model, even in the context of blocked design fMRI. Better model fit will reduce error variance, thereby increasing the sensitivity of the analysis.

\section{Acknowledgment}

This work was funded by the Wellcome Trust.

\section{References}

Friston, K.J., Ashburner, J., Frith, C.D., Poline, J.-B., Heather, J.D., Frackowiak, R.S.J., 1995. Spatial registration and normalization of images. Hum. Brain Mapp. 2, 1-25.

Friston, K.J., Josephs, O., Rees, G., Turner, R., 1998. Nonlinear eventrelated responses in fMRI. MRM 39, 41-52.

Friston, K.J., Glaser, D.E., Henson, R.N.A., Kiebel, S., Phillips, C., Ashburner, J., 2002. Classical and Bayesian inference in neuroimaging: applications. NeuroImage 16, 484-512.

Konishi, S., Donaldson, D.I., Buckner, R.L., 2001. Transient activation during block transition. NeuroImage 13, 364-374.

Kucera, H., Francis, W.H., 1967. Computational analysis of present-day American English. Brown Univ. Press, Providence, RI.

Mechelli, A., Friston, K.J., Price, C.J., 2000. The effects of presentation rate during word and pseudoword reading: a comparison of PET and fMRI. J. Cogn. Neurosci. 12 (Suppl. 2), 145-156.

Mechelli, A., Price, C.J., Friston, K.J., 2001. Nonlinear coupling between evoked rCBF and BOLD Signals: a simulation study of hemodynamic responses. NeuroImage 14, 862-872.

Mechelli, A., Price, C.J., Henson, R.N.A., Friston, K.J., 2003. Estimating Efficiency a priori: a comparison of blocked and randomised designs. NeuroImage 18, 798-805.

Price, C.J., Veltman, D.J., Ashburner, J, Josephs, O, Friston, K.J., 1999. The critical relationship between the timing of stimulus presentation and data acquisition in blocked designs with fMRI. NeuroImage 10, 36-44. 